\title{
Reappraisal of progestins in endometriosis therapy
}

\author{
Antti Kauppila \\ Department of Obstetrics and Gynecology, University of Oulu, FIN-90220 Oulu, Finland
}

Endocrine treatment of endometriosis is based upon the suggestion of Kistner that regression of endometriosis can be obtained by induction of pseudo-pregnancy with a combination of oestrogen and progestin (1). The 40-year history of conservative medical therapy of endometriosis demonstrates that many substances and their combinations have been tested, including low-dose oral contraceptives, progestin alone (several candidates), danazol, gestrinone and gonadotrophin-releasing hormone $(\mathrm{GnRH})$ agonist analogues $(2,3)$. Unfortunately, the clinical efficacy of different treatment modalities was investigated mostly retrospectively or in uncontrolled trials until the late $1980 \mathrm{~s}(2,3)$. The more recent studies on the efficacy of drug therapy have been prospective and based on adequate study designs with control therapy and endoscopic confirmation of endometriosis and its behaviour. They have shown that none of the agents or combinations tested so far is superior to others in efficacy. They essentially differ from each other only in tolerance, side effects and cost. Therefore it is still unclear which one of the several currently available alternatives is 'the $\operatorname{drug}(\mathrm{s})$ of choice' for medical treatment of endometriosis with regard to all the aspects that should be taken into account in this context.

Progestin alone was the most popular medical treatment of endometriosis for about 20 years. Danazol was introduced more than 15 years ago, and since then it has widely replaced progestins in clinical practice, although there is no evidence of its superiority over progestational agents. On the contrary, subsequent studies have shown that danazol and medroxyprogesterone acetate (MPA), the most common progestin used in the treatment of this disease, are equally effective in the resolution of endometriosis and pain relief $(4,5)$. Studies from the USA (6) and Italy (7) have also brought evidence that progestin alone produces a good clinical response for endometriosis, which is comparable with the effect of danazol (6). During the past decade, different GnRH agonist analogues have completed the list of drugs inducing antiproliferative effects on endometriosis tissue. Comparisons with danazol have repeatedly failed to reveal any significant difference in their ability to abolish endometriosis implants or to alleviate pelvic pain and other subjective symptoms of endometriosis (3). It is also significant that MPA at doses up to $100 \mathrm{mg}$ a day induces less harmful changes in lipid and lipoprotein metabolism than androgenic danazol. In addition, it is free from hypo-oestrogenic side effects and metabolic sequelae typical of $\mathrm{GnRH}$ analogues. We must further acknowledge that each of the present endocrine treatments is often unable to achieve complete and permanent resolution of endometriosis. Recurrences are common after surgical or medical conservative treatment. The risk of symptomatic recurrence has been as high as $40 \%$ at 9 years follow up (8). Each of the present hormonal treatment modalities, including danazol, GnRH analogues and MPA, is thus far from optimal. Consequently, more efficient and safer compounds are required for the suppressive treatment of endometriosis and the control of endometriosis-associated complaints. There are no breakthrough discoveries in sight that could open up totally new prospects for endometriosis therapy. Therefore more scientific attention should be given to potential agents that have not been tested adequately for treatment of this disease.

Several progestational compounds are interesting in this respect. The most attractive alternatives are nonandrogenic and free from metabolic side effects, e.g. dydrogesterone, cyproterone acetate, and natural progesterone meet these requirements. In fact, in my department we have found that natural oral progesterone is efficacious in the treatment of endometriosis (unpublished data). Previous studies have also shown that a novel progestin, dienogest, does not affect lipid metabolism in humans (9) or rats (10). It thus also belongs to the group of agents that deserve attention from researchers. A study group in Japan has recognized this challenge and presents in this issue of European Journal of Endocrinology interesting and promising results on the efficacy and mechanism of action of dienogest in experimental endometriosis, using the model of renal subcapsular autotransplantation of endometrial tissues in rats (11). In terms of short-term effects, dienogest diminished the volume of endometriosis implants as much as danazol, which has also been a 'gold standard' in clinical trials on endometriosis (3).

The mechanisms by which different hormonal compounds induce their suppressive actions on endometriosis are not known in detail. Possibly both direct and indirect actions are involved. According to the classic theory, suppression of the anterior pituitaryovarian axis activity with concomitant discontinuation of the stimulatory oestrogen support to endometrial tissues is probably the central event in this process. Progesterone and possibly also androgen receptors play 
a significant role in the mediation of the antiproliferative actions of progestational agents directly upon the epithelial and stromal components of endometriosis tissue. To study the importance of this mechanism, the Katsuki group employed RU 486, a progesterone receptor antagonist, in their experiments (11). RU 468 diminished the suppressive action of dienogest on endometrial cells only somewhat, not totally as in the case of danazol. On the basis of these findings, the inhibitory action of dienogest on cell proliferation seems to be dependent on its progestational effect and, in contrast with danazol, also on other actions. These actions were also explored in this study.

The peritoneal environment is disturbed in several ways in patients with endometriosis (for references, see 11). For instance, the number of macrophages and the concentrations of macrophage-induced cytokines and prostaglandin metabolites are increased in the peritoneal fluid of patients with this disease. These and other immunological mediators may be important in the development of endometriosis, and they may interfere with the persistence of subfertility, a typical condition of young patients with endometriosis. Restoration of normal peritoneal fluid characteristics might thus be helpful in the return of fertility. In their comprehensive work, Katsuki and his co-workers observed that dienogest decreased the number of macrophages, diminished the production of interleukin- $1 \beta$ by macrophages and normalized the natural killer activity of mononuclear cells originating from peritoneal fluid and the spleen (11). The extent to which dienogest improves the peritoneal environment may contribute to its clinical efficacy in, for example, pain relief and restoration of fertility.

The effects of dienogest on the intracellular signalling systems were also assessed in the present experiments with and without RU 486, which is known to block progesterone receptor-mediated actions (11). The inhibition of protein kinase A activity and the decreased cyclic AMP content, which are also effects of dienogest and danazol, was due to the progestational activity of the drugs. In contrast with danazol, dienogest also inhibited the protein kinase $C$ activity of rat endometrial cells in the presence of RU 486. This finding substantiates the view that dienogest also induces inhibitory actions by mechanisms other than those due to its progestational properties. The therapeutic significance of this biochemical phenomenon, which is specific to dienogest, is unclear and should be elucidated in clinical conditions.

The results of animal experiments are naturally not directly applicable to humans. Although preliminary data from an uncontrolled trial have shown dienogest to be highly effective for endometriotic lesions and symptoms (8), prospective trials are necessary to learn more of its clinical value in general and its exact relationship to other progestins, danazol and $\mathrm{GnRH}$ analogues. It was of interest that such studies are already going on in several countries (11).
The principles of treating endometriosis have changed noticeably over the past ten years. Nowadays, endoscopic interventions employing resection, ablation, coagulation or vaporization of endometriosis lesions at the time of the initial diagnosis have a central role in the treatment of endometriosis and associated complaints. Other methods are complementary. The observations showing that suppressive endocrine treatment does not improve the prospects of infertile endometriosis patients have further diminished the significance and use of such therapy $(3,6)$. Recent data showing that laparoscopic elimination of minimal or mild endometriosis enhances fecundity of infertile women (12) is in line with this trend.

However, there are still several indications for endocrine treatment of endometriosis. Such therapy administered for 3-6 months postoperatively may enhance the efficacy of the surgical elimination of endometriosis in pelvic pain relief. Short-term observations support this notion (5), but the long-term efficacy of postoperative hormonal therapy has not been evaluated in large-scale prospective studies. Another major indication for endocrine therapy is recurrent endometriosis. Some patients who have developed symptomatic recurrence refuse further surgical interventions, and some patients may need pain relief while waiting for a reoperation. In addition, this treatment alone is practical and adequate in many cases of less troublesome recurrence. Young patients with endometriosis are often referred to an infertility clinic for treatment with in vitro fertilization or other methods of assisted reproduction technology. Initially, many of them are exposed to drugs that induce anterior pituitary-ovarian axis and endometrial suppression, which is a prerequisite for optimal stimulation of ovarian follicles. The symptom-free condition of the endometriosis patient during the waiting period is an additional advantage of this treatment.

To sum up, the present study (11) is a good example of a well accomplished experimental investigation providing useful information for clinicians and researchers interested in the endocrinology of endometriosis and its hormonal therapy. It shows that there is still much to do to clarify the significance of progestins in the treatment of endometriosis in general and per compound. Many progestational formulations, such as dienogest, which are capable of effective suppression of endometrial lesions, merit further evaluation and the attention of clinicians.

\section{References}

1 Kistner RW. The treatment of endometriosis by inducing pseudopregnancy with ovarian hormones. American Journal of Obstetrics and Gynecology 195872 264-278.

2 Schmidt CL. Endometriosis: a reappraisal of pathogenesis and therapy. Fertility and Sterility 198544 157-173.

3 Kauppila A. Changing concepts of medical treatment of endometriosis. Acta Obstetricia et Gynecologica Scandinavia 1993 $72324-336$. 
4 Telimaa S, Puolakka J, Rönnberg L \& Kauppila A. Placebocontrolled comparison of danazol and high-dose medroxyprogesterone acetate in the treatment of endometriosis. Gynecological Endocrinology $1987151-61$.

5 Telimaa S, Rönnberg L \& Kauppila A. Placebo-controlled comparison of danazol and high-dose medroxyprogesterone acetate in the postoperative treatment of endometriosis. Gynecological Endocrinology 19871 363-371.

6 Moghissi KS. Treatment of endometriosis with estrogen-progestin combination and progestin alone. Clinical Obstetrics and Gynecology $198831823-828$.

7 Vercellini P, DeGiorgio O, Oldani S, Cortesi I, Panazza S \& Crosignani PG. Depot medroxyprogesterone acetate versus an oral contraceptive combined with very-low-dose danazol for longterm treatment of pelvic pain associated with endometriosis. American Journal of Obstetrics and Gynecology 1996175 396-401.

8 Wheeler JM \& Malinak LR. Recurrent endometriosis: incidence, management, and prognosis. American Journal of Obstetrics and Gynecology 1983146 247-253.
9 Köhler G, Göretzlehenr G \& Brachmann K. Lipid metabolism during treatment of endometriosis with the progestin dienogest. Acta Obstetricia et Gynecologica Scandinavia 198968 633-635.

10 Katsuki Y, Sasagawa S, Takano Y, Shibutani Y, Aoki D, Udagawa Y et al. Animal studies on endocrinological profile of dienogest, a novel synthetic steroid. Drugs under Experimental Clinical Research 199723 45-62.

11 Katsuki Y, Takano Y, Futamura Y, Shibutani Y, Aoki D, Udagawa Y \& Nozawa S. Effects of dienogest, a synthetic steroid, on experimental endometriosis in rats. European Journal of Endocrinology 1998138 216-226.

12 Marcoux S, Maheux R, Bérubé S, and The Canadian Collaborative Group of Endometriosis. Laparoscopic surgery in infertile women with minimal or mild endometriosis. New England Journal of Medicine 1997337 217-222.

Received 3 October 1997

Accepted 6 October 1997 